\title{
Errors-in-variables problems in transient electromagnetic mineral exploration
}

\author{
K. Lau, J. H. Braslavsky and G. C. Goodwin
}

\begin{abstract}
This paper is motivated by a real estimation problem arising in transient electromagnetic mineral exploration. A specific sub-problem of interest in this area is that of finding the transfer function linking two antennae without utilising the primary excitation. Natural excitation is provided by nearfield and far-field lightning strikes ('sferics'). This estimation problem is surprisingly difficult due to its intrinsic errors-invariables nature. This paper proposes a combined time- and frequency-domain method to address the difficulty and obtain model estimates that are shown to be of practical use for sferics noise cancellation.
\end{abstract}

\section{INTRODUCTION}

Mining companies throughout the world expend significant effort searching for new ore bodies. A variety of technologies are utilised including geological surveys, diamond drilling, and airborne mineral exploration. Our interest here is with ground-based transient electromagnetic (TEM) surveying using an Australian-developed technology known as Geoferret ${ }^{\mathrm{TM}}$ [1]. This system operates in a similar way to metal detectors used for finding lost jewellery on the beach, namely a pulse of current is used to induce a current in the hidden metal. The transient decay of the induced current is detected by measurement coils in the instrument. The system used for mineral exploration is very large with a transmitter loop of the order of a square kilometre and measurement coils of the order of a metre in diameter.

The detection of decaying currents induced in deep (up to $500 \mathrm{~m}$ ) mineral deposits is a problem with very low signalto-noise ratios. Hence, substantial effort is directed at noise reduction techniques. The sources of noise are many and varied and include instrument noise, harmonic disturbances (including $50 \mathrm{~Hz}$ power line interference) and 'sferics' (short for 'atmospherics') — environmental electromagnetic radiation generated by lightning.

Multiple antennae are typically deployed for detection in systems such as Geoferret ${ }^{\mathrm{TM}}$, which raises the possibility of using the sferics seen at one receiving antenna to cancel the sferic component seen at another. In order to do this, it is first necessary to find the inter-antenna transfer function. This leads to an experiment in which one measures the signal at two antennae (with the primary excitation turned off) and then estimates the model between them. We note that in this context, the input to the model is the sferic component of the signal at the reference antenna. The rest of this signal is considered to be 'measurement noise'.

The authors are with the ARC Centre for Complex Dynamic Systems and Control (CDSC), The University of Newcastle, Callaghan, 2308, NSW, AUSTRALIA. \{K.Lau, Julio.Braslavsky, Graham.Goodwin\}@newcastle.edu.au
In carrying out the above program of work, a major problem was observed, namely that the 'input' signal was measured in a very noisy environment. This is a classical errors-in-variables (EIV) problem [2]. The emphasis of the current paper is on the presentation of this EIV estimation problem and its solution.

In Section II we provide background information on TEM surveying and sferics. Then in Section III, we describe the EIV difficulties associated with sferics noise cancellation in TEM surveying with systems such as Geoferret ${ }^{\mathrm{TM}}$. A brief review of EIV is given in Section IV.

In Section $\mathrm{V}$, two approaches are proposed to address the EIV problem. The first (natural) approach is to use filtering to separate the signal from the noise. However, the resultant model is unsatisfactory for noise cancellation. We then use time-domain selection of favourable parts of the input record. This exploits the fact that sferics are highly non-stationary and appear as isolated pulses that stand out from the background noise. As we show in Section V-B the combination of time and frequency selectivity does lead to a model that can be successfully applied to noise cancellation.

\section{BACKGROUND TO TEM SURVEYING AND SFERICS}

\section{A. TEM surveying}

Transient electromagnetic surveying (also known as timedomain electromagnetic surveying) is a technique used in mineral exploration to detect underground conductive ore bodies by induction and detection of electromagnetic (EM) fields. Fig. 1 shows a typical system configuration for TEM surveying with a system such as Geoferret ${ }^{\mathrm{TM}}$.

The typical operation of a TEM surveying system consists of two phases: firstly, the transmission of a primary field, during which no measurements are made, and secondly, after the transmitter is switched off, the detection of the secondary field response (i.e., the transient response) of the earth. To generate the primary field, a pulsed current waveform is passed through a loop or coil of wire (the transmitter), which is laid on the surface of the area to be surveyed. The primary field induces superficial underground eddy currents, which in turn induce eddy currents at greater depths in any conductive ore bodies. These underground decaying currents produce the secondary field, which can be measured by the array of receiving antennae on the earth's surface. The magnitude and rate of decay of the secondary field depends on the electrical conductivity of the ground, and, through posterior signal processing, allows the identification and location of target ore bodies at depths of up to $500 \mathrm{~m}$. 

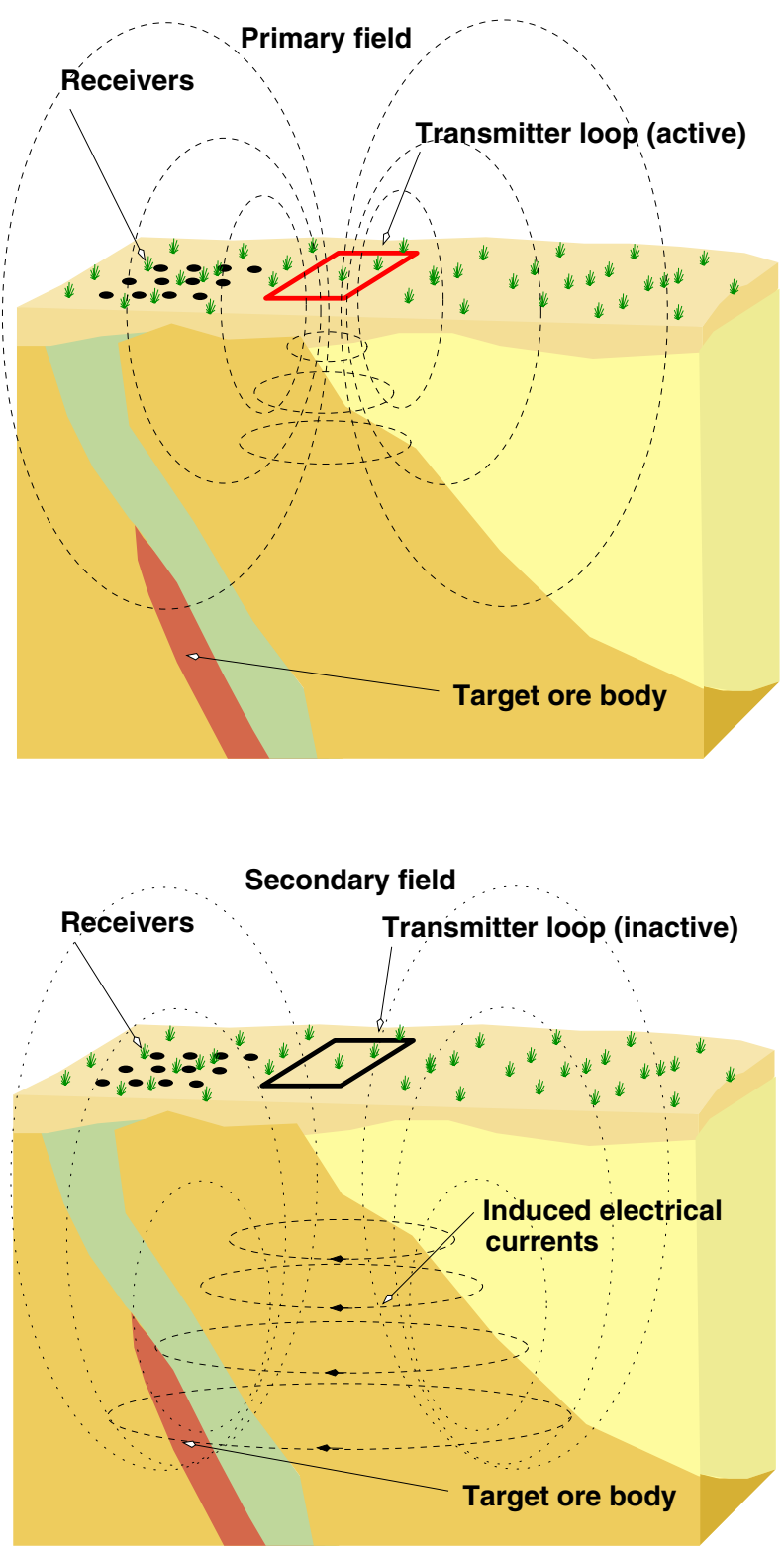

Fig. 1. Typical ground-based TEM surveying system configuration.

We consider a system in which a periodic current waveform consisting of a series of square pulses of alternating sign is used. The secondary field at different locations is measured using a number of receiver coils. The measured data is interpreted to obtain a geological model which is consistent with the data.

A more detailed description of TEM surveying can be found in [3], [4] and [5].

\section{B. Sferics}

A major contributor to noise in the detection of deep underground ore bodies is sferics: environmental EM radiation that dominates receiver instrument noise in some environments (such as close to the equator). Sferics (short for 'atmospherics') originate from the EM radiation produced by lightning strikes that travel thousands of kilometres through the space between the earth and the ionosphere, which acts as a waveguide. Hence, local and distant lightning storms contribute to the sferics noise measured at any single point.

Sferics noise can be divided into two groups, local and distant. Local sferics noise consists of large, infrequent, bursts of short pulses (i.e., it is impulsive), and is nonstationary in nature. Fig. 2 shows a measured large sferic occurring at approximately $0.002 \mathrm{~s}$.

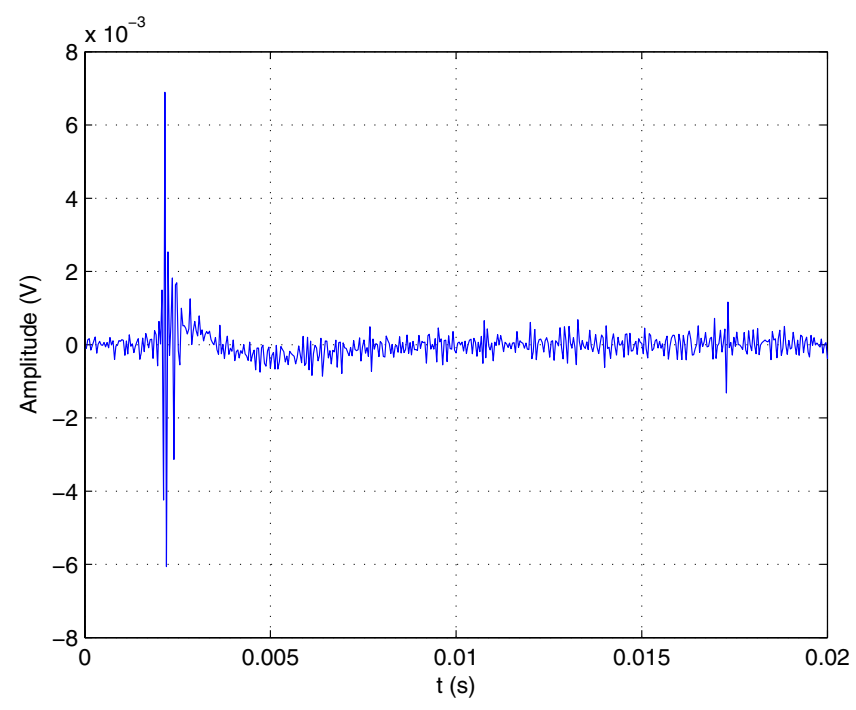

Fig. 2. A measured sferic (output antenna).

Distant sferics noise may be characterised as essentially coloured noise. It has been estimated that there are approximately 100 lightning strikes per second worldwide. Thus, distant sferics noise consists of many small pulses and can be considered to be quasi-stationary. The spectrum of distant sferics noise is concentrated in the $1-500 \mathrm{~Hz}$ and $2.5-10$ $\mathrm{kHz}$ frequency bands. The dip in the spectrum between 500 $\mathrm{Hz}$ and $2.5 \mathrm{kHz}$ is due to the attenuation of the earthionosphere waveguide at these frequencies. Fig. 3, which is reproduced from [6], shows a typical, moderately distant sferics pulse and diagram of the different components of the electromagnetic spectrum. The two sferics frequency bands are clearly labelled.

\section{DifFICULTIES IN SFERICS NOISE CANCELLATION}

Our primary interest in the current paper is in sferics. The goal is to use sferics detected at a reference antenna to carry out sferics noise cancellation at a different, output antenna. We note that related noise cancellation ideas have been used previously in other types of TEM surveying systems, e.g., [7], [8] and [9]. However, these ignore the effects of errorsin-variables.

A preliminary experiment aimed at verifying the veracity of the above idea was performed. A simple model consisting of a gain plus a time shift was fitted to the data (we will later show that this model is a good approximation at low frequencies). However, when the model was utilised for 


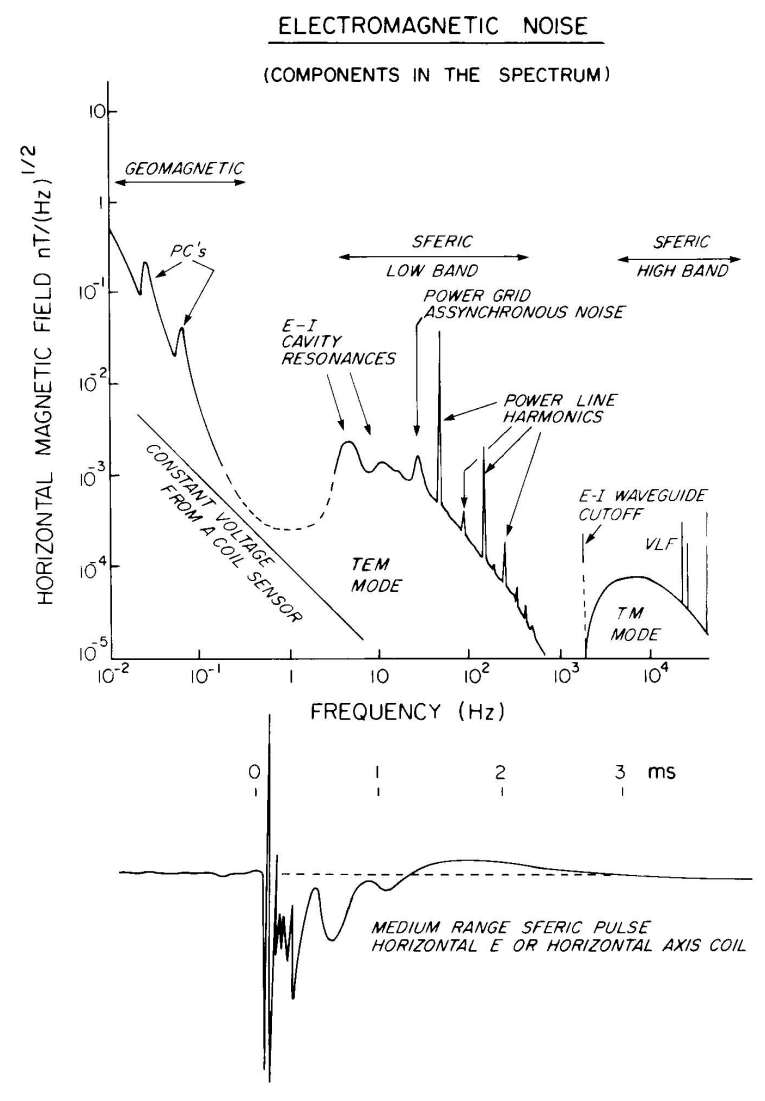

Fig. 3. Components of the electromagnetic spectrum and a moderately distant sferics pulse (reproduced from [6]).

noise cancellation there was no reduction in the noise at the secondary antenna. Fig. 4 illustrates the results. The spectra for the noise at the output antenna before and after performing noise cancellation are shown. The figure also shows the spectrum of the cancellation signal, i.e., the estimate of the sferics noise obtained by using the fitted model and the measurement at the reference antenna. It can be seen that the cancellation signal is significantly smaller than the actual noise and, hence, the spectrum of the residual is almost identical to the measured noise.

Careful examination of the problem reveals that the measured signal at the reference antenna is corrupted by significant 'measurement noise'. (Of course one does not 'know' the incoming sferics; one can only infer it from measurements). This suggests that there is an errors-invariables problem. We shall see in Section V that this is indeed the case and that both time- and frequency-domain 'selectivity' are necessary to mitigate the difficulty.

\section{BACKGROUND TO ERRORS-IN-VARIABLES}

The presence of measurement noise on the input has implications for system identification. In particular, the noise on the input results in biased estimates of the model. Indeed, if the measurement noise on the input is large at some frequency, then the gain of the system will appear to be small at that frequency. Hence, the identification algorithm

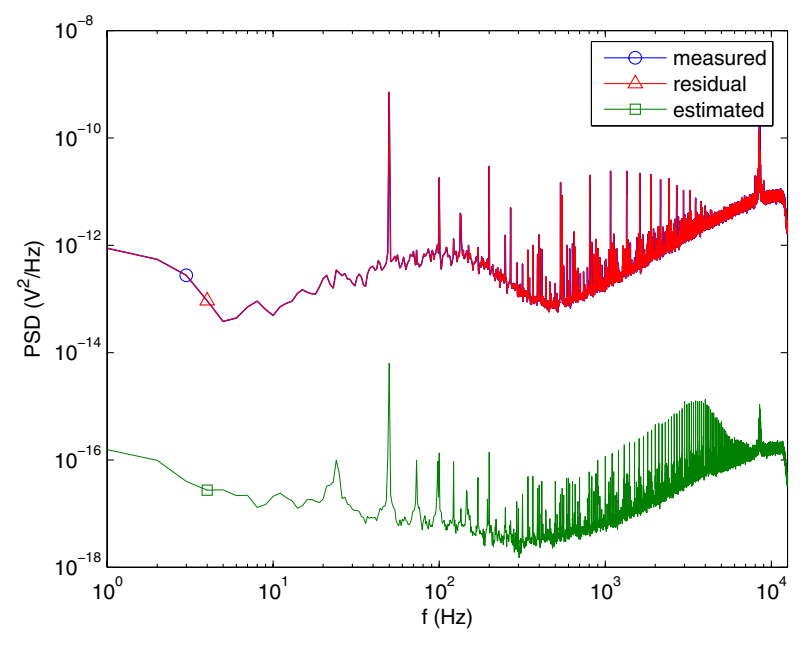

Fig. 4. Spectra of the measured noise, residual noise after performing noise cancellation, and the cancellation signal (estimated sferics noise). Model fitted to raw input-output data. Note that the spectra for the measured and residual noise are almost identical.

will tend to under-estimate the gain at that frequency, as is illustrated by the following simple example.

Example 1: Suppose the input $u_{0}(k)$ and output $y_{0}(k)$ of a scalar system are related as follows:

$$
y_{0}(k)=g_{0} u_{0}(k)
$$

i.e., $y_{0}(k)$ is simply a scaled version of $u_{0}(k)$. We assume that $u_{0}(k)$ is a random signal with zero mean and a variance of $\operatorname{Var}\left\{u_{0}\right\}$.

We wish to obtain an estimate $\hat{g}$ of $g_{0}$ from $N$ noisy measurements of $u_{0}(k)$ and $y_{0}(k)$. Let the $k$ th pair of measurements be denoted by $u(k)$ and $y(k)$. We have

$$
\begin{aligned}
& u(k)=u_{0}(k)+\eta_{u}(k), \\
& y(k)=y_{0}(k)+\eta_{y}(k),
\end{aligned}
$$

where $\eta_{u}$ and $\eta_{y}$ are assumed to be zero-mean, uncorrelated, white noise sequences with variances $\operatorname{Var}\left\{\eta_{u}\right\}$ and $\operatorname{Var}\left\{\eta_{y}\right\}$, respectively. It is also assumed that $\eta_{u}$ and $\eta_{y}$ are uncorrelated with $u_{0}$.

If we ignore the noise on $u(k)$, we can use a least squares estimator to find $\hat{g}$. The least squares fit for $g_{0}$ is given by

$$
\hat{g}=\frac{\sum_{k=1}^{N} y(k) u(k)}{\sum_{k=1}^{N} u^{2}(k)} .
$$

It can be seen that as the number of measurements $N \rightarrow \infty$,

$$
\hat{g} \rightarrow \frac{1 / N \sum_{k=1}^{N}\left(g_{0} u_{0}(k)+\eta_{y}(k)\right)\left(u_{0}(k)+\eta_{u}(k)\right)}{1 / N \sum_{k=1}^{N}\left(u_{0}(k)+\eta_{u}(k)\right)^{2}}
$$

and hence, since $u_{0}, \eta_{y}$ and $\eta_{u}$ are independent, that

$$
\hat{g} \rightarrow \lambda g_{0}, \quad \text { where } \lambda=\frac{\operatorname{Var}\left\{u_{0}\right\}}{\operatorname{Var}\left\{u_{0}\right\}+\operatorname{Var}\left\{\eta_{u}\right\}} .
$$


We observe the following:

- If $\eta_{u}(k)$ is zero (no measurement noise on the input), then $\hat{g} \rightarrow g_{0}$ as $N \rightarrow \infty$, i.e., it is consistent.

- If there is measurement noise on the input $(\operatorname{Var}\{u\} \neq$ $0)$, then $\hat{g} \rightarrow \lambda g_{0}$, where $0<\lambda<1$. Hence, the least squares estimate is asymptotically biased. As might be expected, the bias is less significant when the SNR of $u$ is large $\left(\operatorname{Var}\left\{\eta_{u}\right\} / \operatorname{Var}\left\{u_{0}\right\}\right.$ small). We also observe that the least squares estimator under-estimates the gain.

- If $\operatorname{Var}\left\{\eta_{u}\right\} / \operatorname{Var}\left\{u_{0}\right\}$ is known, then $\lambda$ is known and hence, in theory, we can correct the bias (by dividing by $\lambda$ ). However, this approach can lead to large errors in the estimate, particularly when $\lambda$ is small. We note that this occurs when the SNR of $u$ is small.

The preceding simple example demonstrates the importance of taking into account EIV when performing system identification.

The study of EIV is a well established research area. Recent surveys are given in [2] and [10]. Early work on the topic includes [11] and [12]. More recent work includes [13]-[17].

One of the recurring themes in the EIV literature is that, one can obtain a unique estimate provided that additional prior information is available. Examples are specific relationships between polynomial orders and coprimeness of polynomials (e.g., [13], [15]).

However, these methods tend to be intrinsically sensitive to the assumptions since one has to effectively 'lift' the true input out of the measured noisy input. Indeed, if the measurement noise is (relatively) large, then one is trying to extract a small signal from a large noise background by utilising subtle differences in signal properties. It follows that if one has a poor signal to noise ratio, then this is unlikely to be a robust procedure.

Returning to the sferics noise cancellation problem of Section III, it was found that the signal to noise ratio is very poor. Consequently, great care is needed to be able to successfully carry out identification.

\section{OVERCOMING ERRORS-IN-VARIABLES PROBLEMS IN SFERICS NOISE CANCELLATION}

We now show that despite the low signal to noise ratio (SNR) of the measured reference signal, we are able to fit a useful model to the data by utilising 'time and frequency selectivity' to improve the SNR.

Fig. 4 in Section III shows the measured power spectral density (PSD) for the output antenna. We observe that there is a large noise mound between approximately 20 and 400 $\mathrm{Hz}$. In this section, we focus on reducing this mound for the following reasons:

- It is known from the literature (see Section II-B) that sferics have significant power in this frequency band.

- We are particularly interested in the cancellation of noise at low frequencies.

We concentrate on the reduction of the 'baseline' noise spectrum. We are not concerned with the harmonic components as these latter components can be removed separately.
For our purpose, the rest of spectrum, i.e., the harmonic disturbances and the parts of the spectrum above approximately $400 \mathrm{~Hz}$ and below approximately $20 \mathrm{~Hz}$ are treated as measurement noise. These components are largely due to instrument noise and harmonic disturbances (including $50 \mathrm{~Hz}$ power line interference). Other notable features of the spectrum include the peak at $8 \mathrm{kHz}$, which is due to the resonance of the antenna coil, and the low frequency component $(<10 \mathrm{~Hz})$, which is in part due to small movements of the antenna relative to the (static) magnetic field of the earth.

We can verify that medium- to near-field sferics make a significant contribution to the noise between 20 and 400 $\mathrm{Hz}$ by viewing a filtered version of the signal. Fig. 5 shows a filtered version of the sferic shown in Fig. 2. The corresponding filtered signal from the reference antenna is also shown. We note that these signals were produced by using a non-causal filter to remove the components below 20 $\mathrm{Hz}$ and above $400 \mathrm{~Hz}$ as well as the harmonic components.

From Fig. 5 it is clear that, in the 20 to $400 \mathrm{~Hz}$ frequency band, the sferics pulse is large relative to the measurement noise. It can also be seen that there is a high degree of correlation between the output and reference antennae. Furthermore, a gain plus a time shift can be used to model the relationship between these signals. It should be noted that the output can be delayed relative to the reference, or vice versa. In the following sections, we fit models of this form (gain plus a time shift) to the data.

\section{A. Frequency-domain selectivity (filtering)}

As discussed above, Fig. 5 suggests the use of a gain plus a time shift to model the correlation between the reference and output nodes. A natural first approach is to use least squares to fit a model to the filtered signals. We note that this approach uses frequency-domain selectivity (filtering) to improve the SNR of the input.

Figs. 6 and 7 illustrate the results when this approach is taken. In this case, $5 \mathrm{~s}$ of filtered data were used to fit the model, and a separate set of data was used to validate the model. The filtered output signal $z_{f}$ and the estimate of this signal $\hat{z}_{f}$ (obtained by applying the model to the filtered reference signal) are shown in Fig. 6. The residual $z_{f}-\hat{z}_{f}$ is also shown. It can be seen that the gain is under-estimated. As discussed in Section IV this is expected when there is noise on the input measurement.

Fig. 7 shows the residual spectrum when the estimate $\hat{z}_{f}$ is used for noise cancellation. The measured spectrum is also shown. Recall that the aim is to reduce the baseline noise between 20 and $400 \mathrm{~Hz}$. However, it can be seen that there is little or no reduction in the total amount of noise. Whilst there is a slight reduction in the noise at the centre of the mound, this is counteracted by an increase at other frequencies. The increase in the noise at some frequencies is due to the presence of the input noise in the cancellation signal.

We conclude that in this case, the noise on the input is detrimental for two reasons. Firstly, because it results 
in under-estimation of the gain, and secondly, because the cancellation signal contains an extra component due to the input noise. This extra component increases the noise at the output when the noise cancellation is performed.

\section{B. Combined frequency- and time-domain selectivity}

The filtered sferics noise has a non-stationary characteristic as it consists of large pulses separated by sections of coloured 'measurement noise'. This suggests that we can obtain an input with a higher SNR by selecting only the large pulses for model fitting and noise cancellation. We do this by setting the parts of the signal between the pulses to zero (i.e., by windowing sections of the data). We refer to this process as 'marking' the input. For an example of a marked signal see the estimated signal in Fig. 9.

Fig. 8 shows the spectrum of the reference signal and the spectrum of the filtered and marked version of the signal. It can be seen that the marking process appears to separate the sferics noise mound from the other noise sources. We note that the estimated SNR of the unmarked signal is between 0.3 and 1 , depending on where the line is drawn between near- and far-field sferics. The SNR of the marked part of the signal is between approximately 60 and 100 .

Figs. 9 and 10 illustrate the results when the test in Section V-A is repeated using the marked version of the (same) data to fit the model and generate the cancellation signal. The filtered output $z_{f}$, estimated output $\hat{z}_{f}$ and residual error $z_{f}-\hat{z}_{f}$ are shown in Fig. 9. It can be seen that the fit for the large sferics pulses is very good.

Fig. 10 shows the measured and residual spectra when the estimate $\hat{z}_{f}$ is used for noise cancellation. The sferics noise mound is now significantly reduced.

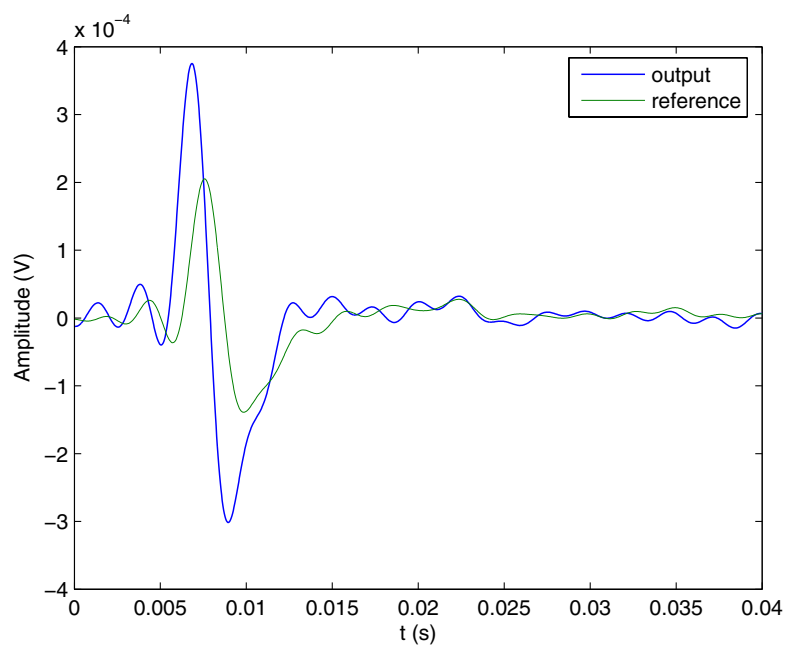

Fig. 5. Filtered sferic pulse at the output and reference antennae.
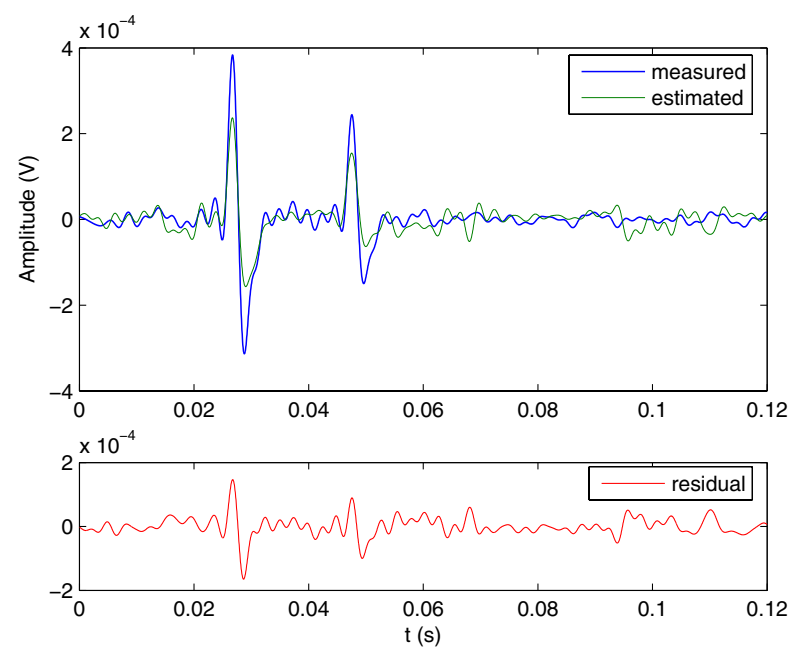

Fig. 6. Filtered output $z_{f}$, estimated output $\hat{z}_{f}$ and residual error $z_{f}-\hat{z}_{f}$. Model fitted using the entire filtered input and output.

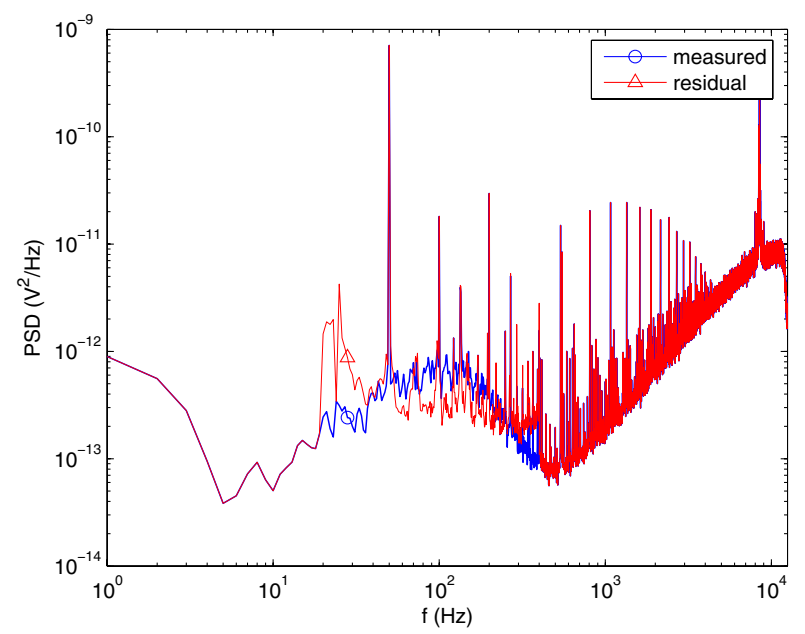

Fig. 7. Spectra before and after noise cancellation. Model fitted using the entire filtered input and output.

\section{CONCLUSIONS}

This paper has described an errors-in-variables problem encountered in a mineral exploration application. This application is characterised by a very poor signal to noise ratio on the input rendering the usual methods for obtaining a model inappropriate. By exploiting both time and frequency selectivity, it has been shown that a suitable model can be obtained. The veracity of this estimated model has been tested by using it for noise cancellation on an independent (validation) data set. This was shown to produce a significant reduction in the noise. 


\section{ACKNOWLEDGEMENTS}

We would like to acknowledge J. Lee, M. Downey, R. Turner and A. Maddever, from BHP-Billiton for their contribution to this project. We especially thank J. Lee for proposing the problem and M. Downey for collecting the experimental data used in this paper.

\section{REFERENCES}

[1] R. A. Carter. Exploration by the numbers. Engineering and Mining Journal, 206(5):34-38, 2005.

[2] T. Söderström. Errors-in-variables methods in system identifaction. In Preprints of the 14th IFAC symposium in System Identification, Newcastle, Australia, 2006.

[3] P. Kearney, M. Brooks, and I. Hill. An introduction to geophysical exploration. Blackwell Science, Malden MA, 3rd edition, 2002.

[4] Geophysical exploration for engineering and environmental investigations, 1998. Technical engineering and design guides as adapted from the U.S. Army Corps of Engineers No. 23. ASCE Press.

[5] M. N. Nabighian and J. C. Macnae. Time domain electromagnetic prospecting methods in electromagnetic methods. In M. N. Nabighian, editor, Electromagnetic Methods in Applied Geophysics Vol. 2, Application, Part A, pages 427-520. Society of Exploration Geophysics, 1991.

[6] J. C. Macnae, Y. Lamontagne, and G. F. West. Noise processing techniques for time-domain EM systems. Geophysics, 49:934-48, July 1984

[7] G. Buselli, H. S. Hwang, and J. P. Pik. AEM noise reduction with remote referencing. Exploration Geophysics, 1998.

[8] A. Stephan and K.-M. Strack. A simple approach to improve the S/N ratio for TEM data using multiple receivers. Geophysics, 56(6):86369, June 1991.

[9] B. R. Spies. Local noise prediction filtering for central induction transient electromagnetic sounding. Geophysics, 53(8):1068-89, August 1988.

[10] J. C. Agüero and G. C. Goodwin. Identifiability of errors in variables dynamic systems. To appear, Automatica.

[11] R. J. Adcock. Note on the methods of least squares. The Analyst, 4(6):183-184, 1877.

[12] R. Frisch. Statistical confluence analysis by means of complete regression systems. Pub. No 5, University of Oslo, Economics Institute, 1934.

[13] P. Stoica and A. Nehorai. On the uniqueness of prediction error models for systems with noisy input-output data. Automatica, 23(4):541-543, 1987.

[14] J. K. Tugnait. Stochastic system identification with noisy input using cumulant statistics. IEEE Transactions on Automatic Control, 37(4):476-485, 1992.

[15] E. Nowak. Identifiability in multivariable dynamic linear errors-invariables models. Journal of the American Statistical Association, 87(419):714-723, 1992.

[16] R. Pintelon and J. Schoukens. System Identification: A frequency domain approach. IEEE Press, 2001.

[17] B. D. O. Anderson, M. Deistler, and W. Scherrer. Solution set properties for static errors-in-variables problems. Automatica, 32(7):1031$1035,1996$.

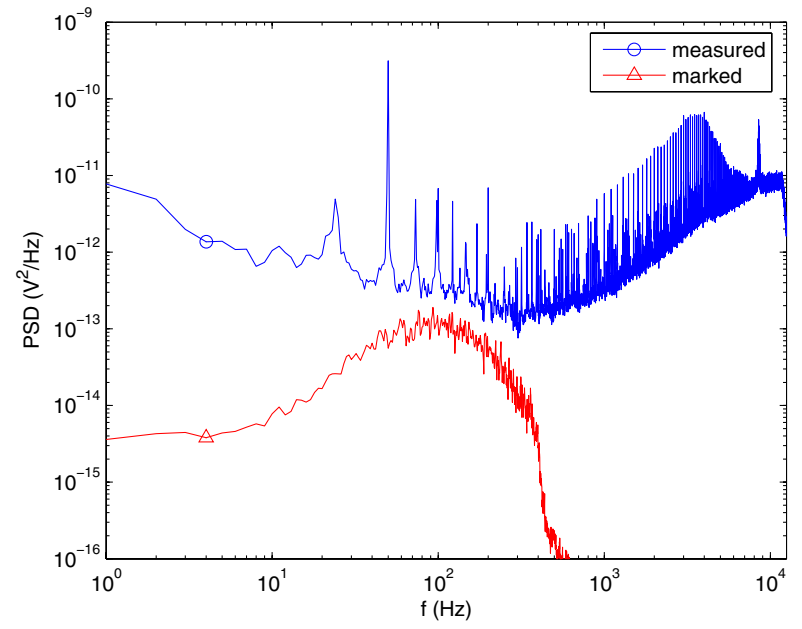

Fig. 8. Spectra of the measured and marked signals for the reference antenna.
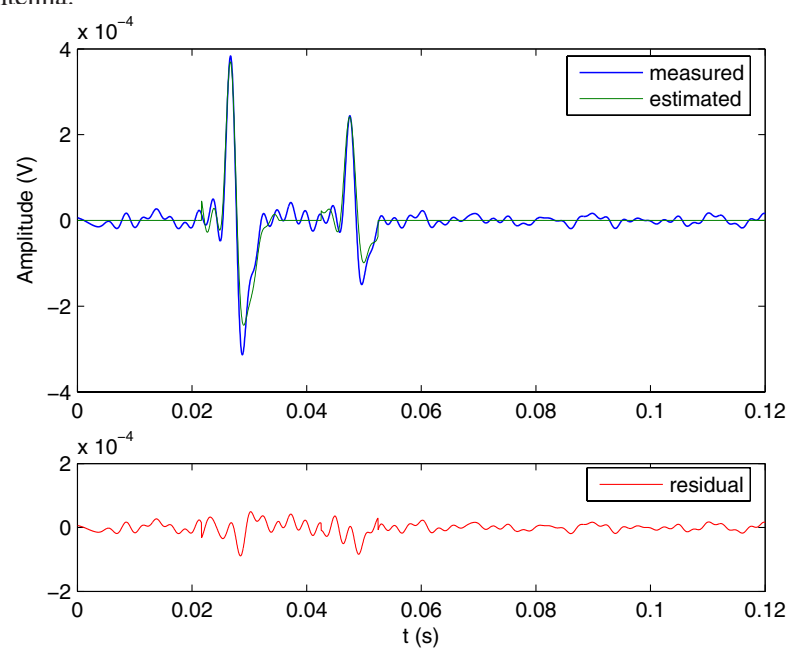

Fig. 9. Filtered output $z_{f}$, estimated output $\hat{z}_{f}$ and residual error $z_{f}-\hat{z}_{f}$. Model fitted using only selected intervals of the filtered input and output.

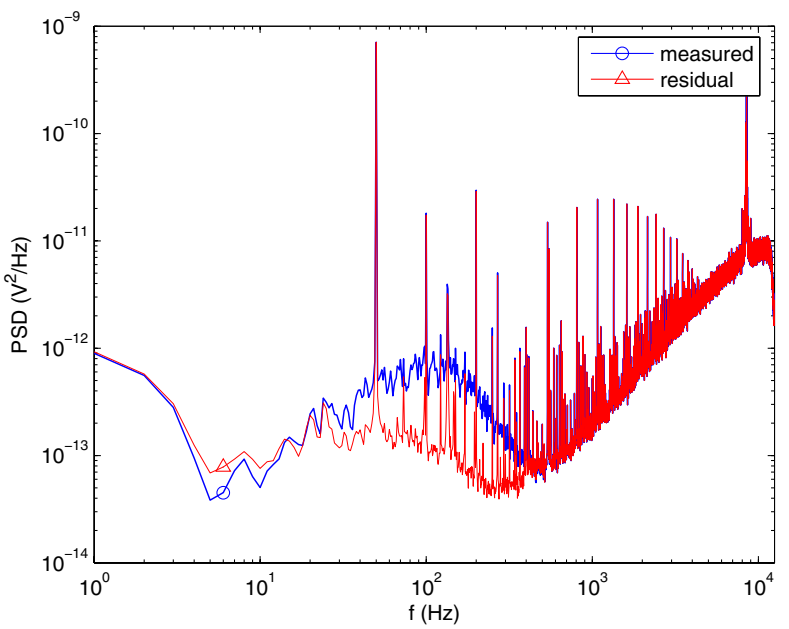

Fig. 10. Spectra before and after noise cancellation. Model fitted using only selected intervals of the filtered input and output. 